\title{
Croissance, indice de consommation et composition corporelle de poulets des races Fayoumi, Rhode-Island et de leur croisement selon deux taux protéiques de la ration
}

\author{
M. ABOU-EL-KASSEM ABD-EL-LATIF (1), A. BORDAS et P. MÉRAT \\ I.N.R.A., Laboratoire de Génétique factorielle \\ Centre National de Recherches Zootechniques, F 78350 Jouy-en-Josas
}

\section{Résumé}

La croissance, l'efficacité alimentaire et la composition corporelle ont été comparées pour des poulets Fayoumi, Rhode-Island (R.I.R.) et $F_{1}$ (Fayoumi $\times$ R.I.R. et R.I.R. $\times$ Fayoumi) répartis en 2 lots recevant respectivement une ration à 14,5 et à 20 p. 100 de protéines totales. Les 2 croisements sont nettement supérieurs à la moyenne des 2 races parentales pour la croissance pondérale et l'un d'eux $\left(0^{*}\right.$ Fayoumi $\times$ ㅇ R.I.R.) dépasse le parent le plus lourd (R.I.R.). L'hétérosis paraît plus marquée pour la croissance avec le régime le moins riche en protéines totales et il en est de même pour l'indice de consommation. D'autre part, l'absence d'interaction entre type génétique et régime pour la plupart des variables montre en particulier que la Fayoumi n'est pas avantagée comparativement aux autres génotypes par l'utilisation d'un régime pauvre en protéines. Pour la composition corporelle, le poulet Fayoumi, malgré sa croissance médiocre, a un rendement à l'abattage (p. 100 éviscéré / vif) qui n'est pas inférieur à celui des poulets Rhode-Island et des croisements.

Mots clés : Poulet, Fayoumi, taux protéique, croissance, composition corporelle.

\section{Summary}

Growth, feed efficiency and body composition of chicks from the Fayoumi and Rhode-Island red breeds and their cross with two different protein levels

Growth rate, feed efficiency and body composition were compared for Fayoumi, RhodeIsland (R.I.R.) and $F_{1}($ Fayoumi $\times$ R.I.R. and R.I.R. $\times$ Fayoumi) chicks distributed in 2 groups receiving respectively a ration with 14.5 and 20 per cent total protein. The 2 crosses are appreciably superior to the average of the 2 parental breeds for growth rate and one of them $($ Fayoumi $\times$ R.I.R.) is superior to the heavier parent (R.I.R.). The heterosis for growth seems more marked with the 14.5 p. 100 protein feed, and for feed efficiency the tendency is the same.

The lack of interaction between genetic type and feed for most traits shows in particular that the Fayoumi is not at a relative advantage when given a low-protein ration. On the other hand, the Fayoumi chick, in spite of its poor growth rate, is not inferior to the R.I.R. and $F_{1}$ chicks for slaughter yield.

Keys words : Broiler, Fayoumi, protein level, growth, body composition.

(1) Adresse permanente : Faculté d'Agriculture, Université d'Assiout, Egypte. 


\section{Introduction}

L'effet du taux protéique du régime sur les performances de croissance (taux plus élevé donnant des poulets plus lourds et moins gras) a été revu, par exemple par Gahrlich \& Brake (1980), Hulan et al. (1980), Hulan \& Proudfoot (1981), TouchbuRn et al. (1981). Du point de vue génétique, certains auteurs ont comparé l'effet du niveau protéique de la ration sur différentes lignées, croisements ou génotypes. Sorensen (1980) indique, après 7 générations de sélection de 2 lignées sur la vitesse de croissance, l'une en présence d'un aliment standard et l'autre avec un aliment à taux protéique réduit, qu'il n'y avait pas au total de différence significative de gain de poids jusqu'à l'âge de 6 semaines entre ces lignées, mais que pour le poids à 6 semaines, on observait une interaction lignée $\times$ taux protéique importante. Hulan \& Proudfoot (1981) observent des interactions entre lignées et régimes de taux protéiques différents distribués à plusieurs âges, pour le poids à 21,49 ou 70 jours. En Egypte, AbDel-Hamid et al. (1978) ont trouvé que la croissance et l'efficacité alimentaire étaient améliorées par un régime riche en protéines, à la fois chez la Leghorn et chez la race Dandarawy.

La race Fayoumi, répandue en Egypte, s'est révélée avoir une efficacité alimentaire inférieure aux normes courantes pour la production d'œufs en batteries avec un aliment standard à 16 p. 100 de protéines totales. L'efficacité de ses croisements $F_{1}$ avec une lignée expérimentale Rhode-Island était plus élevée (Mérat et al., 1983). En phase de croissance, nous n'avons pas connaissance de données comparatives sur l'efficacité alimentaire des mêmes types génétiques. D'autre part, la Fayoumi a pu avoir à s'adapter à des rations suboptimales, notamment quant au niveau protéique, et il nous a paru intéressant de mesurer son efficacité alimentaire en présence d'un taux protéique relativement bas. Tel est l'objet du présent travail.

\section{Matériel et méthodes}

\section{A. Animaux et conditions expérimentales}

Le 20 mars 1983 une éclosion pedigree, correspondant à une ponte de 3 semaines, comprenait des poussins Fayoumi, Fayoumi $\times$ Rhode-Island (R.I.R.), R.I.R. $\times$ Fayoumi et R.I.R. Cette éclosion était issue de 9 pères Fayoumi accouplés à 27 mères Fayoumi et 27 mères R.I.R., et 9 pères R.I.R. accouplés à 27 mères Fayoumi et 27 mères R.I.R. La descendance comprenait ainsi les 2 lignées parentales et leurs 2 croisements réciproques. Les poussins mâles, sexés à la naissance, étaient gardés pour la présente expérience. De l'éclosion à 4 semaines d'âge, tous étaient élevés au sol dans une même cellule chauffée. L'aliment (à 18 p. 100 de protéines totales et $2800 \mathrm{kcal} / \mathrm{kg}$ d'énergie métabolisable, voisin d'un aliment «démarrage 》 pour poules pondeuses) et l'eau étaient distribués ad libitum. La durée d'éclairement était de 24 heures pendant la première semaine et de 10 heures ensuite.

A l'âge de 4 semaines, 30 mâles par type génétique, soit 120 au total, étaient choisis au hasard dans les familles les plus nombreuses et mis en cages individuelles 
dans un local maintenu à $28 \pm 1{ }^{\circ} \mathrm{C}$. La moitié des poulets de chaque type génétique recevait ad libitum un aliment à 14,5 p. 100 de protéines totales (régime 1), l'autre moitié un aliment à 20 p. 100 de protéines (régime 2). La répartition des types génétiques et des régimes était égalisée selon l'étage de la batterie. Les 2 régimes avaient la même énergie métabolisable $(2700 \mathrm{kcal} / \mathrm{kg})$ et la même teneur estimée (4.8 p. 100) en cellulose brute. La composition des régimes est donnée au tableau 1.

\section{TABleau 1}

Composition des 2 régimes.

Composition of the 2 rations.

\begin{tabular}{|c|c|c|}
\hline Composants & $\begin{array}{l}\text { Régime } 1 \\
(14,5 \text { p. } 100 \\
\text { de protéines })\end{array}$ & $\begin{array}{l}\text { Régime } 2 \\
(20 \text { p. } 1.00 \\
\text { de protéines })\end{array}$ \\
\hline 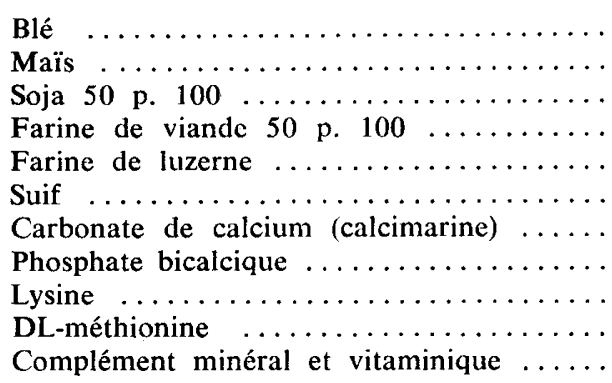 & $\begin{array}{l}40 \\
38 \\
6 \\
1,5 \\
10 \\
0 \\
1,47 \\
0,94 \\
0,24 \\
0 \\
1,8\end{array}$ & $\begin{array}{l}40 \\
25,8 \\
13,3 \\
8 \\
10 \\
0,9 \\
0,02 \\
0 \\
0,03 \\
0,15 \\
1,8\end{array}$ \\
\hline \multicolumn{3}{|l|}{$\begin{array}{l}\text { Teneur calculée } \\
\text { pour les principaux éléments : }\end{array}$} \\
\hline $\begin{array}{l}\text { Energie métabolisable }(\mathrm{kcal} / \mathrm{kg}) \\
\begin{array}{l}\text { Matières azotées totales }(\mathrm{p} .100) \\
\text { Matè }\end{array} \ldots \ldots \ldots \ldots \ldots \\
\text { Matières grasses } \ldots \ldots \ldots \ldots \ldots \ldots \ldots \ldots \ldots \ldots \ldots \\
\text { Cellulose brute } \ldots \ldots \ldots \ldots \ldots \ldots \ldots \ldots \ldots\end{array}$ & $\begin{array}{r}2715 \\
14,5 \\
2,9 \\
4,8\end{array}$ & $\begin{array}{r}2695 \\
20,0 \\
2,9 \\
4,8\end{array}$ \\
\hline
\end{tabular}

Les mesures de composition corporelle, quant à elles, ont porté sur un échantillon de 64 coquelets, soit 8 pris au hasard par type génétique et régime.

\section{B. Mesures et analyses statistiques}

\section{Croissance, indice de consommation et paramètres physiologiques}

Chaque individu était pesé à $5,6,8,10$ et 11 semaines d'âge. Les gains de poids, la consommation alimentaire et l'indice de consommation (aliment consommé / gain de poids) étaient déterminés entre les âges indiqués ci-dessus. Le taux d'acide urique plasmatique était mesuré à 11 semaines, à partir d'une prise de sang faite entre 9 et $11 \mathrm{~h}$ le matin, comme indicateur d'une réponse au taux protéique de la ration (voir p. ex. Featherston, 1969). D'autre part, ce taux a été trouvé relativement élevé dans la race Fayoumi (Mérat et al., 1983). La teneur en eau était déterminée sur 
les fèces récoltées $72 \mathrm{~h}$, des résultats antérieurs (MÉRAT et al., 1983) ayant suggéré chez des pondeuses Fayoumi une valeur faible pour cette teneur.

Une analyse de variance à 2 facteurs (type génétique et régime) était faite, d'après SNedecor \& Cochran (1957). Dans le cas des indices de consommation qui sont des rapports, l'écart vis-à-vis d'une distribution normale n'a pas paru assez important pour invalider cette analyse. D'autre part, les moyennes de chaque variable pour les divers types génétiques étaient comparées deux à deux dans chaque régime par le test de Duncan, d'après STEel \& ToRRIE (1960), après test de l'homogénéité des variances intra-groupes.

Complémentairement, une analyse de covariance à poids corporel fixé a été faite pour l'indice de consommation global de 5 à 11 semaines. Seule la signification de l'effet «génotype » est indiquée (partie «Discussion»).

Enfin, une analyse de variance limitée aux 2 croisements réciproques a été faite pour les critères de croissance et les indices de consommation.

\section{Composition corporelle}

L'abattage avait lieu à 12 semaines. Les coquelets étaient pesés vivants après un jeûne d'environ $16 \mathrm{~h}$. Le poids du sang et celui des plumes étaient déterminés par différence respectivement après saignée et plumage à sec. Les carcasses étaient réfrigérées à $5^{\circ} \mathrm{C}$ jusqu'au moment de la dissection qui avait lieu le surlendemain de l'abattage. Les pattes étaient enlevées et la tête séparée du cou. Ce dernier et les organes de la cavité générale étaient prélevés pour obtenir le poids de la carcasse éviscérée. La graisse abdominale était représentée par le tissu gras entourant le cloaque, le gésier et le ventricule suivant la description de RICARD \& Rouvier (1967). Toutes les mesures étaient exprimées en p. 100 du poids vif. Enfin, la demi-carcasse éviscérée était mise $96 \mathrm{~h}$ à l'étuve à $60^{\circ} \mathrm{C}$ pour déterminer sa teneur en eau.

Les analyses statistiques faites sur l'échantillon utilisé pour les mesures de composition corporelle étaient identiques à celles décrites pour les mesures de croissance et d'efficacité alimentaire.

\section{Résultats et discussion}

\section{A. Croissance et indice de consommation}

Le tableau 2 donne les performances moyennes de croissance et d'indice de consommation, ainsi que le taux d'acide urique plasmatique et la teneur en eau des fèces, par type génétique et régime alimentaire, ainsi que les analyses de variance correspondantes. 


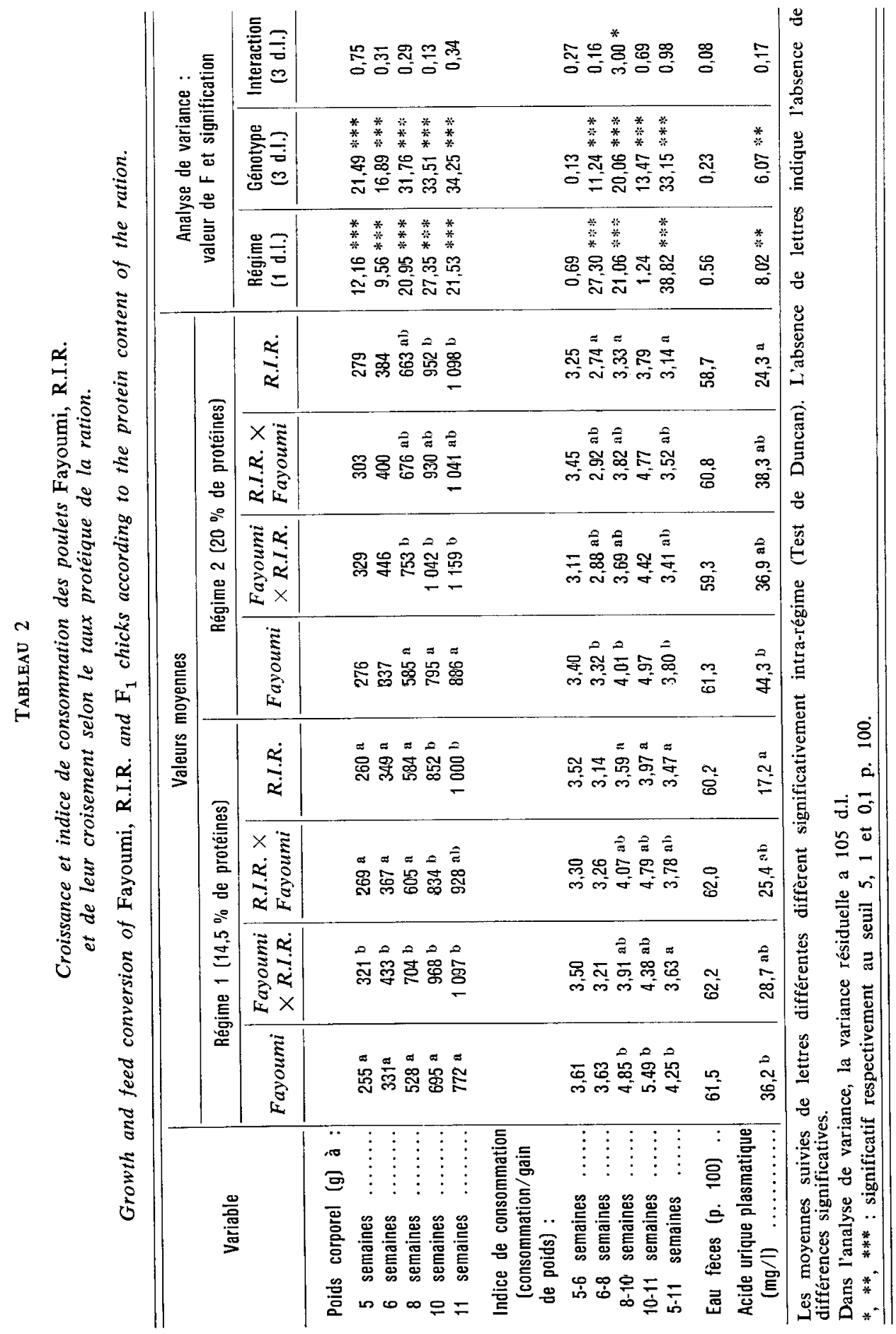


Des différences hautement significatives existent entre génotypes pour toutes les variables sauf l'indice de consommation de 5 à 6 semaines et la teneur en eau des fèces. La croissance pondérale dans chaque régime est la plus faible pour la Fayoumi. Par ailleurs, l'un des croisements dépasse régulièrement les 2 races parentales. Quant à l'indice de consommation, sa valeur plus élevée pour la Fayoumi que pour les autres types génétiques pourrait ne résulter que de la croissance plus lente de la première. Cependant, une analyse de covariance à poids corporel fixé pour l'indice de consommation de 5 à 11 semaines (non présentée en détail) indique que l'effet "type génétique» reste hautement significatif $(P<0,01)$ : il ne reflète donc pas uniquement une différence de croissance pondérale. L'examen des moyennes au tableau 2 indique d'ailleurs que les indices de consommation sont moins bons pour les croisements que pour la R.I.R., quoique l'un de ces croisements ait une croissance meilleure que la $R$ hode. Pour la concentration d'acide urique plasmatique, elle est plus élevée chez la Fayoumi que dans les autres groupes, la R.I.R. a le plus faible taux et les croisements sont intermédiaires. Nous n'avons pas d'interprétation de ces différences.

Ce qui précède montre déjà l'avantage des croisements pour le poids corporel. Nous avons évalué plus précisément au tableau 3 l'hétérosis (écart des croisements à la moyenne des populations parentales) et les effets maternels attribuables à chaque parent selon la décomposition utilisée par BruN \& RouviER (1984).

L'hétérosis est relativement considérable pour le poids à tous âges, si on l'exprime en p. 100 de la moyenne des parents. A partir de 8 semaines, elle paraît un peu plus faible en valeur absolue et en p. 100, avec le régime au taux protéique le plus élevé. Pour les indices de consommation, l'hétérosis, moins importante, est encore nettement marquée en présence du régime le plus «pauvre», mais elle apparaît inexistante avec le régime à 20 p. 100 de protéines, en concordance probable avec l'hétérosis moindre pour le poids.

Entre les croisements réciproques, celui dont le père est de race Fayoumi a le poids le plus élevé (tabl. 2, 3 et 4), ce qui peut être rapproché des résultats d'AMER et al. (1973). On peut supposer que cela provient d'un effet maternel défavorable associé au faible poids d'œuf lorsque la mère est Fayoumi. Pour la croissance, l'écart entre croisements réciproques est aussi important que l'hétérosis. Les 2 croisements diffèrent aussi, quoique relativement moins, pour l'indice de consommation.

Quant à l'effet du régime alimentaire, il est hautement significatif pour le poids corporcl jusqu'à 11 semaines, l'indice de consommation de 6 à 8 et de 8 à 10 semaines, l'indice global de 5 à 11 semaines et le taux d'acide urique plasmatique. L'augmentation du taux d'acide urique avec ce régime concorde avec les données de Featherston (1969) dans lesquelles ce taux est augmenté par une ration à teneur très élevée en protéines totales. Dans l'ensemble, les variables de croissance et d'efficacité sont plus élevées avec le régime le plus riche dans chaque type génétique, et il n'y a pas d'interaction entre type génétique et régime alimentaire, sauf pour l'indice de consommation de 8 à 10 semaines $(P<0,05)$. 


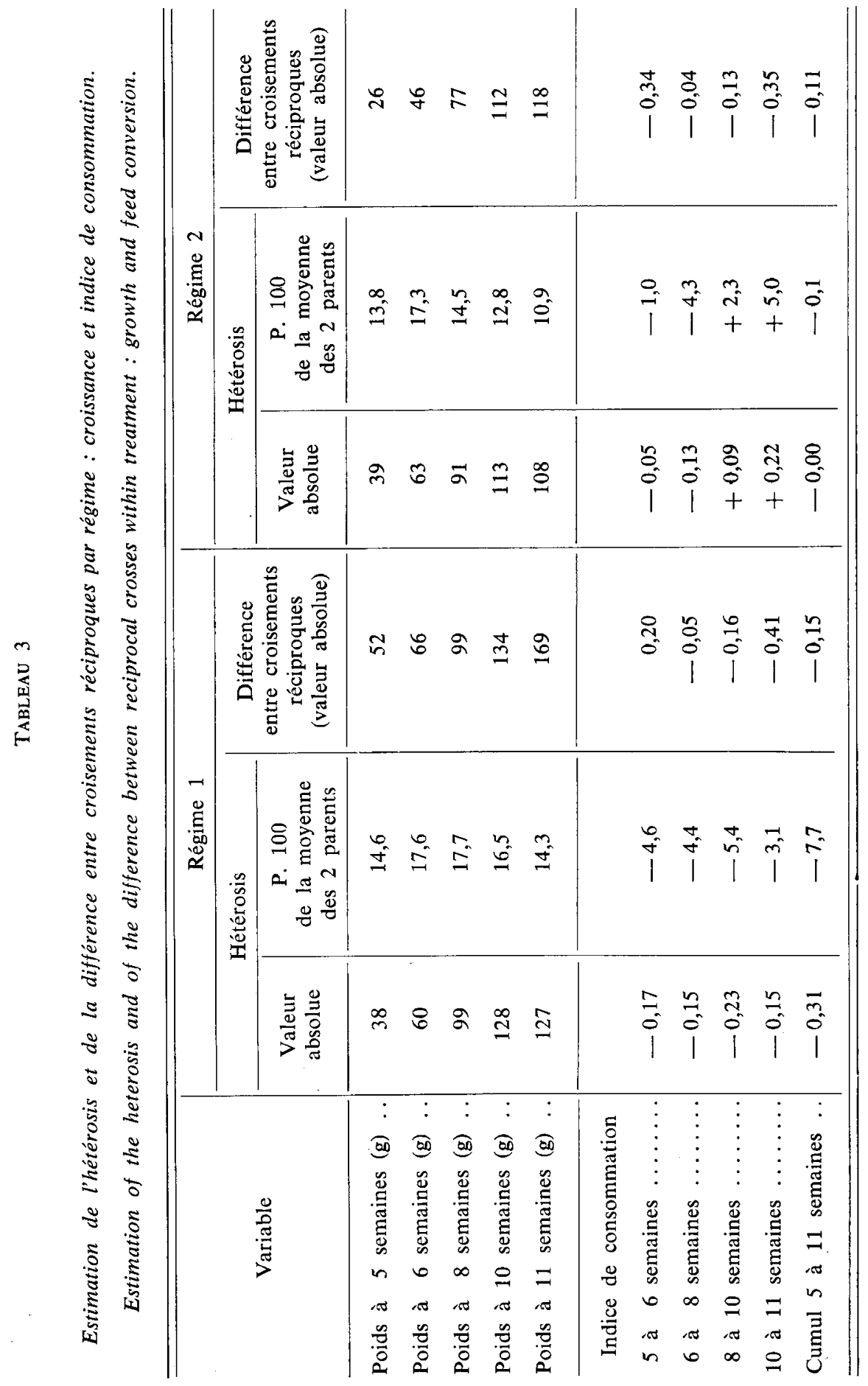




\section{Tableau 4}

Croissance et indice de consommation :
analyse de variance limitée aux 2 croisements réciproques.

Growth and feed conversion : variance analysis limited to the two reciprocal crosses.

\begin{tabular}{|c|c|c|c|}
\hline \multirow{2}{*}{ Variable } & \multicolumn{3}{|c|}{$\begin{array}{l}\text { Analyse de variance : } \\
\text { valeur de } \mathrm{F} \text { et signification }\end{array}$} \\
\hline & $\begin{array}{l}\text { Régime } \\
\text { (1 d.1.) }\end{array}$ & $\begin{array}{l}\text { Génotype } \\
\text { (1 d.l.) }\end{array}$ & $\begin{array}{l}\text { Interaction } \\
\quad(1 \text { d.1.) }\end{array}$ \\
\hline \multicolumn{4}{|l|}{ Poids corporel $(\mathrm{g})$ à } \\
\hline 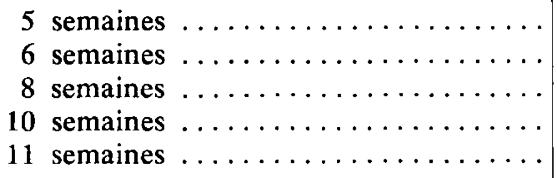 & $\begin{array}{l}5,93 * \\
2,61 \\
12,33 * * * \\
12,22 * * * \\
9,37 * *\end{array}$ & $\begin{array}{l}20,91 * * * \\
15,15 * * * \\
26,45 * * * \\
25,53 * * * \\
25,51 * * *\end{array}$ & $\begin{array}{l}2,15 \\
0,46 \\
0,38 \\
0,21 \\
0,81\end{array}$ \\
\hline \multicolumn{4}{|l|}{ Indice de consommation } \\
\hline 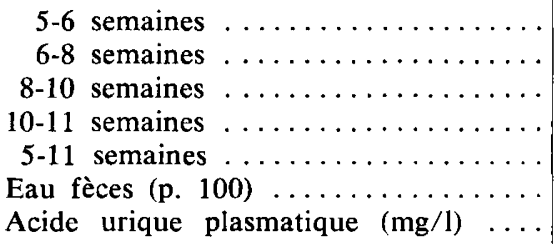 & $\begin{array}{l}0,18 \\
16,38 * * * \\
5,49 * \\
0,00 \\
15,30 * * * \\
0,56 \\
7,49 * * *\end{array}$ & $\begin{array}{l}0,05 \\
0,30 \\
1,95 \\
3,65 \\
4,52 * \\
0,06 \\
0,06\end{array}$ & $\begin{array}{l}0,82 \\
0,01 \\
0,02 \\
0,02 \\
0,10 \\
0,10 \\
0,39\end{array}$ \\
\hline
\end{tabular}

\section{B. Composition corporelle}

Le tableau 5 montre les valeurs moyennes par type génétique et régime, avec l'analyse de variance correspondante, pour chaque variable.

Il n'y a pas de différence significative entre types génétiques pour les pourcentages de sang, foie, gésier et graisse abdominale. Par contre, les pourcentages de plumes, pattes et intestin sont moindres et celui de la tête, de la carcasse éviscérée et la perte d'eau de la demi-carcasse à l'étuve sont plus élevés chez la Fayoumi que dans les autres groupes. On peut penser que la proportion plus élevée de la tête chez la Fayoumi est associée à sa croissance plus lente, mais on ne peut dire la même chose pour les pattes dont le pourcentage est plus faible chez cette race. Les croisements sont toujours des valeurs intermédiaires entre celles des races parentales. Pour le pourcentage de plumes, toutefois, les croisements réciproques accusent une différence dans les 2 régimes, avec la valeur la plus faible pour la $\mathrm{F}_{1}($ Fayoumi $\times$ R.I.R.). Ceci est parallèle à la valeur plus élevée de la race parentale R.I.R. comparée à la Fayoumi pour cette variable, suggérant la possibilité d'un effet lié au sexe. Bien entendu, l'absence de signification de certaines différences peut provenir des effectifs limités. 


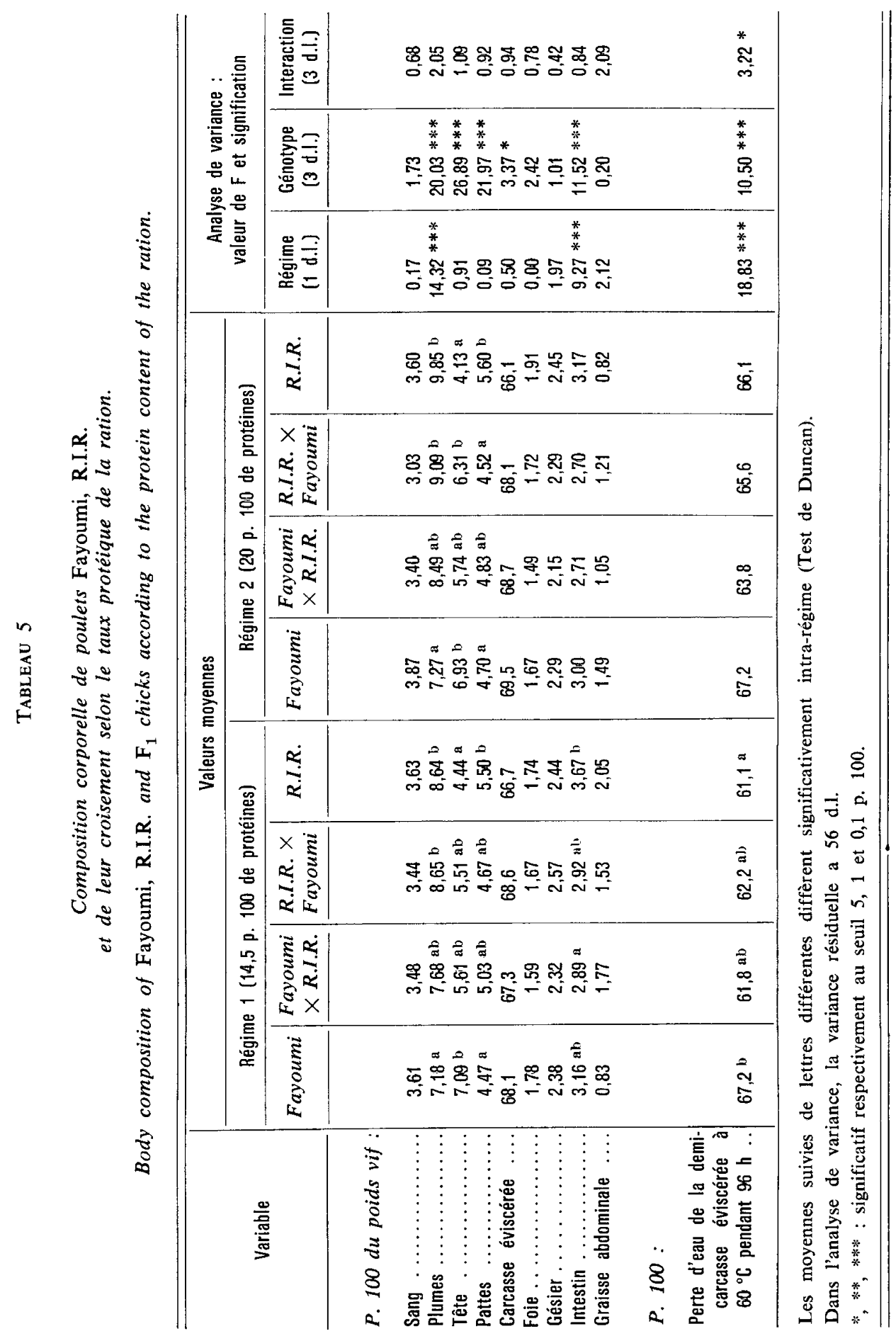


D'autre part, nous n'avons pas actuellement d'interprétation des différences trouvées significatives.

En ce qui concerne le rendement de la carcasse éviscérée, on peut noter aussi que le poulet Fayoumi, malgré sa croissance médiocre, n'est pas inférieur au poulet Rhode-Island et aux croisements, mais leur est, dans l'ensemble, supérieur.

Le tableau 6 estime l'hétérosis pour plusieurs variables de composition corporelle exprimées en p. 100 du poids corporel. Les écarts trouvés sont tous faibles et nous ne les commentons pas en détail. Il en est de même des effets maternels évalués d'après les différences entre croisements réciproques.

\section{TABLEAU 6}

Estimation de l'hétérosis et de la différence entre croisements réciproques par régime : composition corporelle.

Estimation of the heterosis and of the difference between reciprocal crosses within treatment : body composition.

\begin{tabular}{|c|c|c|c|c|}
\hline \multirow[b]{2}{*}{ Variable } & \multicolumn{2}{|c|}{ Régime 1} & \multicolumn{2}{|c|}{ Régime 2} \\
\hline & $\begin{array}{l}\text { Hétérosis } \\
\text { (variables } \\
\text { en p. } 100 \mathrm{du} \\
\text { poids corporel) }\end{array}$ & $\begin{array}{l}\text { Différence } \\
\text { entre } \\
\text { croisements } \\
\text { réciproques }\end{array}$ & Hétérosis & $\begin{array}{l}\text { Différence } \\
\text { entre } \\
\text { croisements } \\
\text { réciproques }\end{array}$ \\
\hline Sang & $-0,16$ & 0,04 & $-0,53$ & $+0,39$ \\
\hline Plumes & $+0,25$ & $-0,97$ & $+0,23$ & $-0,60$ \\
\hline Carcasse éviscérée .... & $+0,5$ & $-1,3$ & $+0,6$ & $+0,6$ \\
\hline Foie & $-0,13$ & $-0,08$ & $-0,19$ & $-0,23$ \\
\hline Gésier & $+0,04$ & $-0,05$ & $-0,15$ & $-\cap, 14$ \\
\hline Intestin . & $-0,51$ & $-0,03$ & $-0,38$ & $+0,0 ?$ \\
\hline Graisse abdominale .. & $+0,21$ & $+0,24$ & $-0,03$ & $-0,16$ \\
\hline
\end{tabular}

Pour l'effet du taux protéique du régime, le pourcentage de plumes, de l'intestin et la perte d'eau de la demi-carcasse sont significativement plus élevés avec l'aliment le plus riche en protéines totales. Concernant le plumage, à teneur élevée en protéines et en particulier en acides aminés soufrés, on peut penser qu'un apport alimentaire plus élevé puisse augmenter sa croissance relative. Parmi les différences entre types génétiques, la teneur en eau plus élevée des carcasses des poulets Fayoumi suggère que ces derniers sont les moins gras, car même si elle n'est pas absolue, la corrélation négative entre teneur en lipides totaux et teneur corporelle en eau est connue (VIGNERon et al., 1971). Il ne s'agit pas toutefois des lipides abdominaux, qui ne diffèrent pas significativement entre groupes génétiques, mais cette absence de signification peut correspondre à la variabilité élevée de ce critère et aux effectifs limités. On peut interpréter dans le même sens la perte d'eau à l'étuve plus élevée, dans l'ensemble, des carcasses des poulets nourris en régime plus riche en protéines; on sait que les poulets recevant une ration enrichie en protéines sont moins gras (cf. par 
exemple, Touchburn et al., 1981 ; Ehinger \& Seeman, 1982). La différence de l'importance relative de l'intestin (dont les lipides ne sont pas séparés) suivant le régime, peut peut-être s'interpréter de même. Pour l'interaction entre le génotype et le régime alimentaire, elle n'existe nulle part, sauf pour la teneur en eau des carcasses (au seuil 5 p. 100). Cette dernière est diminuée par le régime pauvre en protéines surtout pour la R.I.R., un peu moins pour les croisements, et pas pour la Fayoumi, suggérant un effet parallèle sur la teneur en lipides corporels. On peut donc conclure dans la majorité des cas qu'il y a un effet indépendant du génotype d'une part et du régime alimentaire de l'autre sur la qualité de la carcasse.

\title{
IV. Conclusion
}

L'absence d'interaction entre type génétique et régime, pour la plupart des variables, soit de croissance et d'efficacité alimentaire, soit de composition corporelle, montre qu'aucun groupe n'est avantagé par rapport à l'autre lorsqu'on utilise une ration relativement pauvre en protéines : en particulier, la Fayoumi n'en tire pas un meilleur parti.

Les effets génétiques observés, et en particulier l'hétérosis, n'ouvrent pas par eux-mêmes de perspective pratique dans un contexte industriel du fait des faibles performances de croissance des 2 types parentaux. On pourrait suggérer cependant une évaluation analogue de l'hétérosis en remplaçant le parent R.I.R. par une souche lourde de type «chair», pour un contexte d'élevage local.

\author{
Reçu le $1^{\text {er }}$ juillet 1985. \\ Accepté le 11 décembre 1985.
}

\section{Références bibliographiques}

Abdel Hamid A., Khalifa M., AkKad A., Khalil A., 1978. Protein requirements of growing chicks in Egypt. 1. Effect of varying levels of dietary protein on the performance and feed efficiency. Alex. J. of Agric. Res., 26, 273-281.

Amer M., Khishin S., Abdu F., 1973. Hybrid vigor and potence ratio in growth rate and meat production of chickens. College of Agric. Res. Bull. Riyad Univ., 1, 84-95.

BRUN J.M., Rouvier R., 1984. Effets génétiques sur les caractères des portées issues de trois souches de lapins utilisées en croisement. Génét. Sél. Evol., 16, 367-384.

Ehinger F., Seeman G., 1982. Einfluss von Futter, Alter und Geschlecht auf die Mastleistung und die Schlachtkörperqualität von Broilern verschiedener Herkunft. 2. Verfettungsgrad. Archiv. f. Geflügelkd., 46, 177-188.

FEATHERSTON W.R., 1969. Nitrogenous metabolites in the plasma of chicks adapted to high protein diets. Poult. Sci., 48, 646-652.

GaHRLich J.D., Brake J.D., 1980. Rearing single comb White Leghorn pullets on low protein diets. Poult. Sci., 59, 1613 (abstr.).

Hulan H.W., Proudfoot F.G., 1981. The effect of different protein levels in three stage diet system on general performance of chickens reared to rooster weight. Poult. Sci., 60, 172-178. 
Hulan H.W., Proudfoot F.G., Ramey D., MCRae K.B., 1980. Influence of genotype and diet on general performance and incidence of leg abnormalities of commercial broilers reared to rooster weight. Poult. Sci., 59, 748-757.

Mérat P., Bordas A., Rolande L'Hospitalier, Jeanine Protais, Bougon M., 1983. Etude des particularités de la poule Fayoumi. III. Ponte, caractéristiques des œufs, efficacité alimentaire et paramètres physiologiques des poules Fayoumi, Rhode-Island et $F_{1}$ en batteries. Génét. Sél. Evol., 15, 147-166.

Ricard F.H., Rouvier R., 1967. Etude de la composition anatomique du poulet. Ann. Zootech., 16, 23-39.

SNedecor G.W., Cohran W.G., 1957. Statistical methods (5th edition). 593 pp., Iowa State Univ. Press. Ames, Iowa, U.S.A.

Serensen P., 1980. Selection for growth rate in broilers fed on diets with different protein level. European Poult. Conf. Hambourg, Sept. 80, 6, 64-71.

Steel G.D., Torrie J.H., 1960. Principles and procedures of statistics. 481 pp., McGrawHill, London.

Touchburn S., Simon J., Leclerce B., 1981. Evidence of a glucose-insulin unbalance and effect of dietary protein and energy level in chickens selected for high abdominal fat content. J. Nutr., 111, 325-335.

Vigneron P., Baron R., Dauzier L., 1971. Evolution post-natale de la quantité d'eau et de lipides du corps et du grand Psoas chez le lapin. Ann. Biol. Anim. Bioch. Biophys., 11, 669-679. 